\title{
Presentation and Outcome Following Radical Cystectomy in Hispanics with Bladder Cancer
}

\author{
M. Manoharan, R. Ayyathurai, R. de Los Santos, A. M. Nieder, M. S. Soloway \\ Department of Urology, University of Miami, Miller School of Medicine, Miami, Florida, USA
}

\begin{abstract}
Objective: Significant racial and ethnic differences in the epidemiology of bladder cancer (BC) exist. Studies have shown African Americans to have lower incidence of bladder cancer than Caucasians, but higher incidence of invasive BC. Hispanics are the largest minority group in the United States. However, no reported studies on bladder cancer among Hispanics are available to date. As our center is in a unique position to study BC in Hispanic patients we were prompted to assess presentation and outcome of patients undergoing radical cystectomy (RC) for BC.

Materials and Methods: Between January 1992 and May 2006, 448 RC were performed. All relevant data were collected and entered into a database. Patients were categorized by ethnicity as Hispanic and non-Hispanic White. African-American and other minority groups were excluded because of the small number. Comparative analysis of Hispanic and non-Hispanic White patients was performed.

Results: 67 (17\%) patients were Hispanic. Mean follow-up period was $41(\mathrm{SD} \pm 40)$ months. Clinical and pathological data between these two groups were compared. Pre-cystectomy T stage was not significantly different between both groups. However, after RC incidence of $\leq \mathrm{T} 1$ disease in Hispanics was lower (22\%) than Caucasians (37\%). This difference, statistically significant $(\mathrm{P}=0.024)$ indicates that Hispanics who undergo $\mathrm{RC}$ present with higher stage disease. KaplanMeier log rank test indicated a difference in disease free survival and disease specific survival between the two groups but however it did not reach statistical significance $(\log \operatorname{Rank} \mathrm{P}=0.082, \mathrm{P}=0.063)$. No significant difference in overall survival was observed $(\mathrm{P}=0.465)$.

Conclusions: Hispanic patients managed with RC for bladder carcinoma present with higher stage disease.
\end{abstract}

Key words: bladder cancer; cystectomy; Hispanic; Latino

Int Braz J Urol. 2008; 34: 691-8

\section{INTRODUCTION}

Bladder Cancer (BC) is the fifth most commonly diagnosed cancer in the United States. It is estimated that 67,160 men and women were diagnosed with $\mathrm{BC}$ and 13,750 died of this disease in the United States in 2007 (1). The prevalence of BC in the U.S. as of January 1, 2004 was approximately 511,790 (2). Radical cystectomy (RC) is an effective curative option for localized muscle invasive and high-grade urothelial cancer (3).
There is significant gender, geographic, ethnic and racial differences in the epidemiology of BC (4). Non-Hispanic White (NHW) men have the highest incidence of BC in the U.S. (40.5 per 100,000) (2). The incidence in African-American (AA) men was studied extensively and shown to be lower than the NHW (20.3 per 100,000) (2,5-7). Hispanic men have a similar incidence to AA men $(20.2$ per 100,000$)$ and are about one-half the NHW rates. In women, the highest rates were in NHW (10.1 per 100,000). The AA and Hispanic women have an incidence of 7.6 and 
5.5 BC per 100,000 women (2). The incidence is lowest in the Asian population. Although the incidence of BC in NHW population exceeds those of AA population, this trend was not observed as regards mortality (7). It has been reported that AA men and women with $\mathrm{BC}$ have higher disease specific mortality compared to the NHW $(7,8)$. There are many reports available on the incidence, disease presentation and mortality of BC in AA and NHW population (8). However, the U.S. Hispanic population is a large and diverse ethnic group whose BC occurrence has not been well reported. Though marked differences among ethnic and racial groups with $\mathrm{BC}$ have been reported in the United States, to our knowledge no specific reports on $\mathrm{BC}$ in the Hispanic population is available to date $(4,9,10)$.

The Hispanics are heterogeneous groups, which include Caribbean (Cubans, Dominicans and Puerto Ricans) Mexican, Central American and South American with other Latin American groups with Spanish ancestry. The term Hispanics refers to ethnicity rather than any particular race (2). Currently Hispanics are the largest minority group in the United States accounting for 13\% (41 million) of the population. They are estimated to reach 47.8 million (15.5\%) by the year 2010. From 1990 to 2000 , the U.S. Hispanic population grew by $58 \%$, while the total U.S. population grew by $13 \%$ (4). More than half of the Hispanic population in the U.S. lives in California, Texas, Florida, and New Mexico.

Miami-Dade County in Florida is the county in U.S. with the largest Hispanic population. Majority of this population with $\mathrm{BC}$ requiring radical cystectomy (RC) were treated at the University of Miami. This places our center in a unique position to study $\mathrm{BC}$ in Hispanic patients. In this study, our aim was to analyze and compare the presentation and outcome of bladder cancer in Hispanic patients who underwent $\mathrm{RC}$.

\section{MATERIALS AND METHODS}

The study was approved by the Institutional Review Board. Between January 1992 and May 2006, $448 \mathrm{RC}$ were performed by a single surgeon. All patients underwent radical cystectomy and bilateral pelvic lymph node dissection as a standard procedure. A retrospective chart review was completed. Demographic, clinical, and pathologic data were entered into a database. Patients were categorized by ethnicity as Hispanic, NHW, AA, and others. A comparative analysis of Hispanic and NHW patients was performed. AA and other minority group patients were excluded because of the small number in our series. Patients with unknown ethnicity and who underwent salvage cystectomy were also excluded from the analyses.

U.S. Census Bureau defines Hispanics as, are those people who speak Spanish as their first language and originated from the Spanish-speaking countries of Central or South America (11). All data on race and ethnicity was self reported. The chi-square and Student's-t-test were employed to study the difference among the ethnic groups. Kaplan-Meier log rank test was used to compare the rates of disease recurrence, overall and disease specific survival between the Hispanic and NHW groups.

\section{RESULTS}

A total of $448 \mathrm{RC}$ were performed by a single surgical team between 1992 and 2006. Twenty-one $(4.6 \%)$ salvage procedures and three $(0.6 \%)$ patients with unknown ethnicity were excluded from the analysis. Twelve $(2.7 \%)$ AA and $12(2.7 \%)$ of other racial minorities were excluded from the analysis. This left 400 remaining patients in a study cohort of which, 333 (83\%) were NHW and 67 (17\%) Hispanics.

A comparative analysis was performed between 67 (17\%) Hispanic patients and 333 (82\%) NHW patients who met the inclusion criteria. The study cohort included $322(81 \%)$ men and $78(19 \%)$ women. The clinicopathological characteristics of the study groups are shown in Table-1. The Hispanic group was significantly younger than the NHW group. $\mathrm{T}$ stage at transurethral resection of bladder tumor (TURBT) was compared in both groups, no difference was found ( $\mathrm{P}=0.789$; Table-2).

The distribution of pathological $\mathrm{T}$ and $\mathrm{N}$ stage at cystectomy were significantly different between the two groups. The incidence of muscle invasive $\mathrm{BC}$ was 
Bladder Cancer in Hispanics

Table 1 -Descriptive details for the Hispanic and non-Hispanic white patients.

\begin{tabular}{lccc}
\hline Characteristics & Non-Hispanics White & Hispanic & p Value \\
\hline Number (n) & $333(83)$ & $67(17)$ & \\
Gender (M:F) & $4: 1$ & $3: 1$ & 0.315 \\
Mean age at RC (years) & 70 & 67 & 0.040 \\
Mean follow-up (months) & 42 & 39 & 0.668 \\
Smokers & $218(65)$ & $43(64)$ & 0.873 \\
Neo-adjuvant chemotherapy & $42(12)$ & $5(7)$ & 0.300 \\
Pathological stage & & & \\
$\leq$ T1 & $124(38)$ & $15(22)$ & \\
T2 & $84(25)$ & $14(21)$ & \\
T3 & $93(28)$ & $30(45)$ & 0.008 \\
T4 & $32(9)$ & $8(12)$ & \\
Node positive & & $2(3)$ & 0.026 \\
$\quad$ N1 & $25(8)$ & $16(24)$ & \\
$\quad$ N2 & $41(12)$ & & \\
Histological grade & & $60(90)$ & 0.130 \\
$\quad$ High & $266(80)$ & $7(10)$ & 0.032 \\
Low & $65(20)$ & $25(37)$ & 0.118 \\
Recurrence & $80(24)$ & $17(25)$ & 0.459 \\
Adjuvant chemotherapy & $56(16)$ & $20(15)$ & 0.594 \\
Bacille Calmette-Guerin & $115(85)$ & $35(52)$ & 0.081 \\
Overall death & $161(48)$ & $17(25)$ & \\
Disease specific death & $54(16)$ & & \\
\hline
\end{tabular}

Values are numbers and () are percentage.

much higher at $78 \%$ in the Hispanic group compared with $63 \%$ in NHW group ( $\mathrm{P}=0.024$; Table-3). In both groups interval between last TURBT date and cystectomy date were compared to identify any delay in performing $\mathrm{RC}$, no difference was observed $(\mathrm{P}>$ $0.05)$.

The 5 year estimated overall, recurrence free and disease specific survival for the entire cohort was
$50 \%, 62 \%$ and $76 \%$ respectively. The 5 year overall survival was not significantly different from the NHW group $(\mathrm{P}=0.465$; Figure- 1$)$. The recurrence free survival of the Hispanic group was significantly lower compared to the NHW group ( $\mathrm{P}=0.082$; Figure-2). Similarly the 5 year disease specific survival of the Hispanic group was lower at $65 \%$ compared to $79 \%$ in the NHW group $(\mathrm{P}=0.063$; Figure-3). There was

Table 2 - T stage before Radical Cystectomy in Hispanic and non-Hispanic white patients.

\begin{tabular}{lccc}
\hline Characteristics & Non-Hispanics White & Hispanic & p Value \\
\hline Clinical stage & & & \\
$\leq \mathrm{T} 1$ & $92(28)$ & $21(32)$ & \\
$\mathrm{T} 2$ & $240(72)$ & $45(68)$ & 0.550 \\
\hline
\end{tabular}

Values are numbers and () are percentages. 
Table 3 - Pathological T stage at Radical Cystectomy in Hispanic and non-Hispanic white patients.

\begin{tabular}{lccc}
\hline Characteristics & Non-Hispanics White & Hispanic & p Value \\
\hline Pathological stage & & & \\
$\leq \mathrm{T} 1$ & $124(37)$ & $15(23)$ & \\
$\mathrm{T} 2,3,4$ & $209(63)$ & $52(78)$ & 0.024 \\
\hline
\end{tabular}

Values are numbers and () are percentages.

no statistically significant difference in frequency of neoadjuvant and adjuvant chemotherapy administration among the study groups. In addition, instillation of Bacille Calmette-Guerin (BCG) vaccine was analyzed and no difference was found between both groups $(\mathrm{P}=0.459)$.

\section{COMMENTS}

In 1999, the Institute of Medicine recommended that greater emphasis should be given to the ethnic groups, including cultural and behavioral attitudes rather than on biologic differences associ- ated with race (12). Subsequently, several reports on the incidence and mortality of cancer among Hispanic men and women were published $(4,9,10)$. These studies have shown significant variations in cancer incidence among different race and ethnicity. Hispanics had lower incidence of lung, breast and prostate cancers compared to NHW or AA. On the other hand, stomach and liver cancers were more common in Hispanics compared to NHW. Myeloma and cervical cancers were significantly more common in Hispanic women (4). These reports have clearly shown that the Hispanic population had an overall lower incidence of cancer than NHW or AA population $(4,10)$.

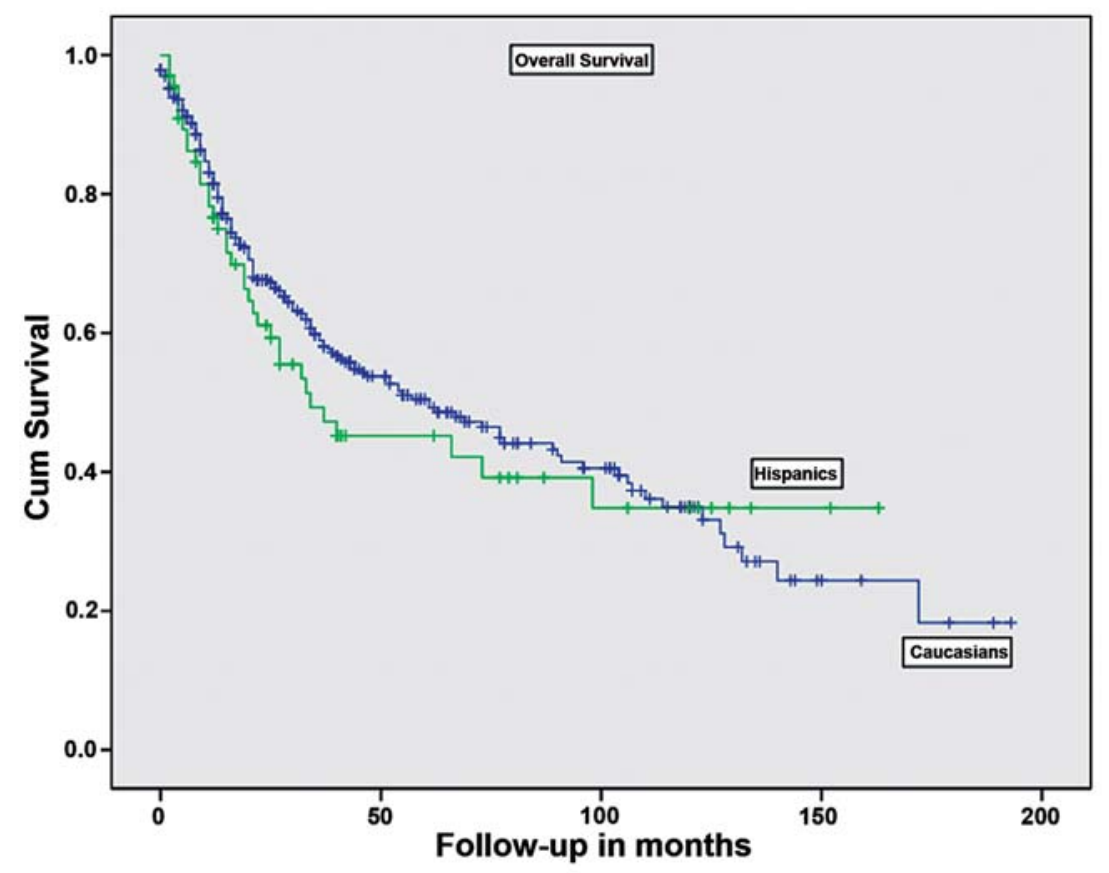

Figure 1 - Overall survival. 


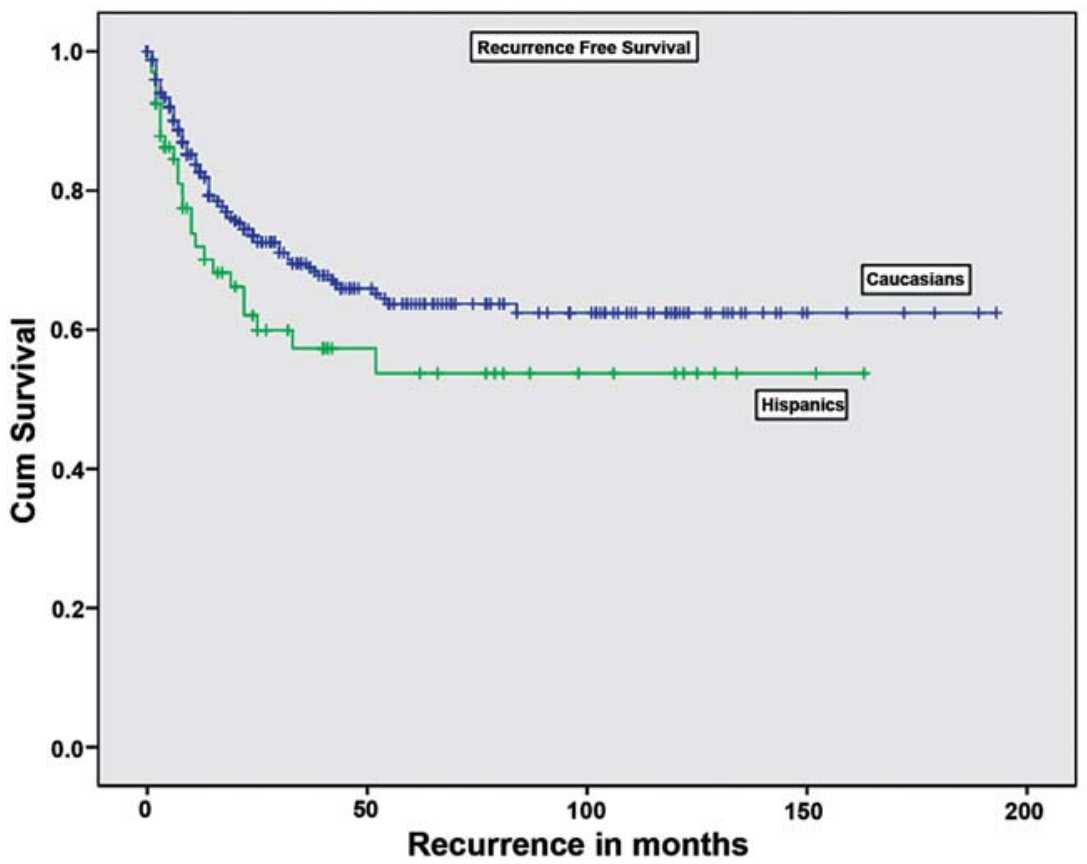

Figure 2-Recurrence-free survival.

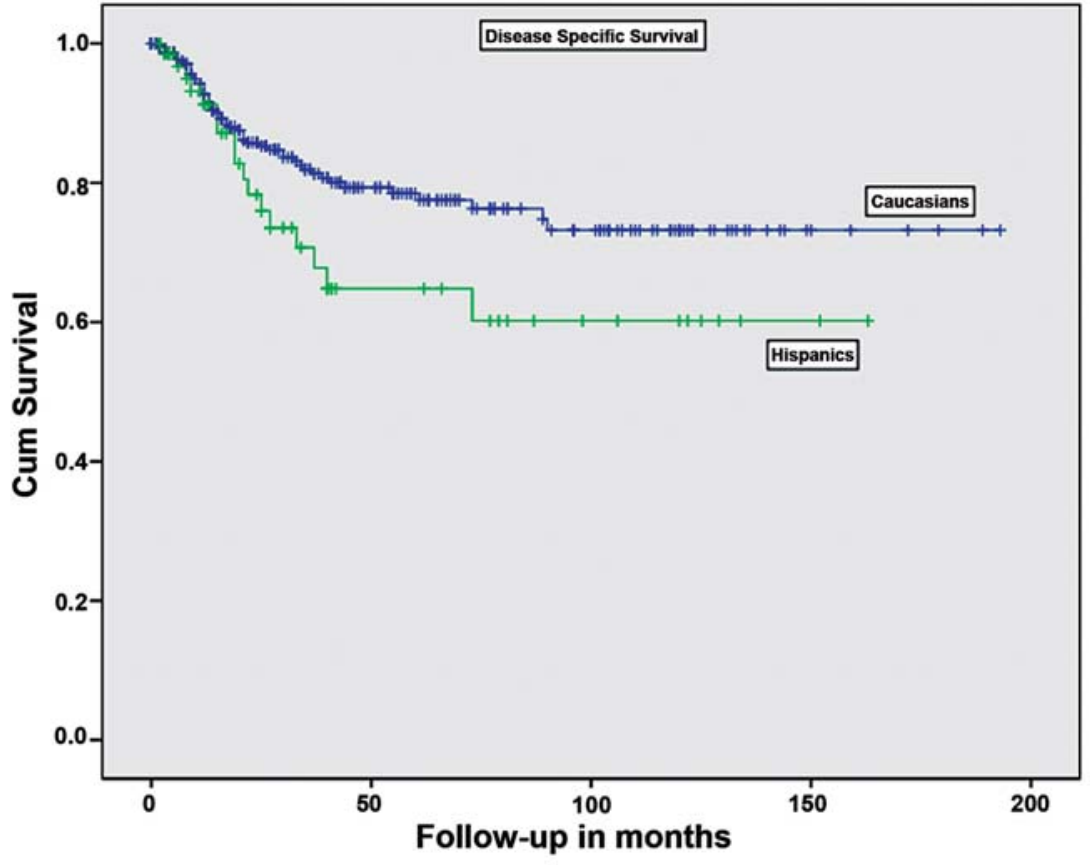

Figure 3 - Disease-specific survival. 
In the U.S., BC is the fourth most common cancer of men in NHW and Hispanics. The exceptions are AA men and Asian Pacific Islanders where the BC is fifth and sixth most common cancer. In women, BC is the tenth most common cancer in NHW and ranked $13^{\text {th }}$ in Hispanic, $14^{\text {th }}$ in AA and $15^{\text {th }}$ in API women (4).

Overall, Hispanic men and women had a lower incidence of $\mathrm{BC}$. The standardized risk ratio for $\mathrm{BC}$ among Hispanic men was 0.74 compared to NHW men (10). However, our results showed that Hispanic men with $\mathrm{BC}$ tended to have a higher stage disease. The number of muscle invasive cancers (stage $\geq \mathrm{T} 2$ ) was significantly higher in the Hispanic group. A total of $78 \%$ of Hispanic patients had muscle invasive cancers compared to $63 \%$ of NHW patients $(\mathrm{P}=0.024)$. Similarly, lymph node involvement of cancer was higher at $27 \%$ in Hispanic patients compared to $20 \%$ in NHW patients. Moreover, $23 \%$ of Hispanic patients were staged N2 compared to $12 \%$ in NHW patients $(\mathrm{P}=0.026)$. Similarly, more Hispanic patients received adjuvant chemotherapy compared to NHW patients; however, this was not statistically significant. Among the $34 \%$ of patients who received BCG treatment, no significance was in fact reached when comparing both groups.

Comparisons between clinical and pathological stage among both groups raised an important question; why are Hispanics presenting with more aggressive disease at radical cystectomy than NHW? When muscle-invasion before and after RC among ethnicities was analyzed, a difference was found in $\mathrm{T}$ stage. The possible explanations for these results might include first, incomplete resection before RC; second Hispanics may have more aggressive disease with faster tumor doubling time and/or; third, delays in the performance of $\mathrm{RC}$ might play a role in higher T stage at cystectomy. Our study showed no significance when the interval between last TURBT date and $\mathrm{RC}$ date were analyzed, possibly excluding the latter explanation.

In the Hispanic group, $37 \%$ patients had recurrences during the follow-up compared to $24 \%$ patients in NHW group. The Hispanic group had higher incidence of local recurrence (54\%) compared to $(43 \%)$ in NHW group $(\mathrm{P}=0.355)$.

Similarly, the 5 year estimated disease specific and recurrence free survival was lower in the Hispanic group, close to statistical significance, which may well be achieved with more follow-up. The advanced $\mathrm{T}$ and $\mathrm{N}$ stage at RC may explain the poor survival outcome in these patients. Although, the 5 year overall survival of the Hispanic population $(45 \%)$ was slightly lower compared to NHW patients (51\%), the difference was not close to the level of statistical significance.

Studies have shown similar disease presentation among AA population and high mortality rate despite low incidence of BC. This difference in survival between AA and NHW populations was attributed to the fact that a larger proportion of NHW cancers are diagnosed at an early stage, and amenable to treatment. However, to date there has been no reported study suggesting higher stage presentation among the Hispanic population with BC.

We further examined the potential causes for higher stage disease among Hispanic patients at RC. Studies on migrated population have demonstrated that environmental factors dominate the epidemiology of many cancers. Wilkinson et al. showed that the overall cancer trends among men in south Florida reported a higher number of Hispanic men with cancers compared to NHW men during the period 1990-98. This study showed the proportion of Hispanic men who presented with cancer had increased nearly $20 \%$ between the past two decades. This change could be attributed to longer residence in the U.S. and presumably more acculturation. With the similar living environment, culture, food habits and exposure to known and unknown carcinogens may eventually equalize the cancer risk of Hispanics compared to that of native born NHW (10). However, we think the fastest growing Hispanic population in the south Florida could have influenced this outcome.

Smoking is a clear risk factor for the incidence and progression of BC. In our study, the smoking pattern was not different between the two ethnic groups. Factors such as cultural attitudes and beliefs were also attributed to be better predictors of advanced disease stage at the time of diagnosis (13). It has been reported that Hispanics fear cancer more than any other diagnosis (14). Socioeconomic status, access to cancer prevention and control services such as cancer screening and health education activities may contribute to differences in both incidence and 
mortality. Many authors have cited cultural and language barriers as explanations for the advanced stage of cancer at the time of diagnosis for Hispanics and other minority groups compared with NHW group (10). The county poverty rate could be a useful indicator of the availability and accessibility to health services. Access to state of the art, quality cancer care is known to be unequal and to exacerbate existing disparities in cancer outcomes.

Several limitations of this study deserve mentioning. This is a retrospective study, which has its own limitations as regards applicability to the general population. Direct and self reporting of race/ ethnicity is preferred, however there are reports showing inconsistent reporting in medical records (15). We did not study the socioeconomic status and other issues such as access to the health care and the pattern of referral from primary care physicians. These could be potential confounding factors in this study.

\section{CONCLUSION}

The purpose of this preliminary study was to provide an overview of presentation and outcome of BC in Hispanic patients after RC. Hispanic patients are at higher risk of having higher stage disease at $\mathrm{RC}$, compared to NHW. The disease specific survival of Hispanic patients after RC was lower compared to NHW. The rationale of these findings may be complex and multifactorial. However, this study showed a considerable difference in the presentation and outcome among the Hispanic patients. Further large population multicenter studies are necessary to validate this finding.

\section{ACKNOWLEDGEMENTS}

Financial support from "CURED 2007" and Mr. Vincent A. Rodriguez.

\section{CONFLICT OF INTEREST}

None declared

\section{REFERENCES}

1. Jemal A, Siegel R, Ward E, Hao Y, Xu J, Murray T, et al.: Cancer statistics, 2008. CA Cancer J Clin 2008; 58: 71-96.

2. Ries LAG, Melbert D, Krapcho M, Mariotto A, Miller BA, Feuer EJ, et al.: SEER Cancer Statistics Review, 1975-2004, National Cancer Institute. Bethesda, MD, http://seer.cancer.gov/csr/1975 2004/, based on November 2006 SEER data submission, posted to the SEER web site, 2007.

3. Ghoneim MA, el-Mekresh MM, el-Baz MA, el-Attar IA, Ashamallah A: Radical cystectomy for carcinoma of the bladder: critical evaluation of the results in 1,026 cases. J Urol. 1997; 158: 393-9.

4. Howe HL, Wu X, Ries LA, Cokkinides V, Ahmed F, Jemal A, et al.: Annual report to the nation on the status of cancer, 1975-2003, featuring cancer among U.S. Hispanic/Latino populations. Cancer. 2006; 107: 1711-42.

5. Aben KK, Kiemeney LA: Epidemiology of bladder cancer. Eur Urol. 1999; 36: 660-72.

6. Kirkali Z, Chan T, Manoharan M, Algaba F, Busch C, Cheng L, et al.: Bladder cancer: epidemiology, staging and grading, and diagnosis. Urology. 2005; 66(6 Suppl 1): 4-34.

7. Miller BA, Kolonel LN, Bernstein L, Young, Jr. JL, Swanson GM, West D, Key CR, Liff JM, Glover CS, Alexander GA, et al. (eds). Racial/Ethnic Patterns of Cancer in the United States 1988-1992, National Cancer Institute. NIH Pub. No. 96-4104. Bethesda, MD, 1996. Available at: http://seer.cancer.gov/publications/ethnicity

8. Fleshner NE, Herr HW, Stewart AK, Murphy GP, Mettlin C, Menck HR: The National Cancer Data Base report on bladder carcinoma. The American College of Surgeons Commission on Cancer and the American Cancer Society. Cancer. 1996; 78: 1505-13.

9. Wilkinson JD, Wohler-Torres B, Trapido E, Fleming LE, MacKinnon J, Peace S: Cancer among Hispanic women in South Florida: an 18-year assessment: a report from the Florida Cancer Data System. Cancer. 2002; 95: 1752-8.

10. Wilkinson JD, Wohler-Torres B, Trapido E, Fleming LE, MacKinnon J, Voti L, et al.: Cancer trends among Hispanic men in South Florida, 1981-1998. Cancer. 2002; 94: 1183-90.

11. The Hispanic Population in the United States. www. census.gov, U.S.C. Bureau, Editor. 2000. Available at: http://www.census.gov/population/socdemo/hispanic/ p20-535/p20-535.pdf 
12. Haynes, M.A. and B.D. Smedley, The Unequal Burden of Cancer: An Assessment of NIH Research and Programs for Ethnic Minorities and the Medically Underserved, Institute of Medicine. Washington, DC. National Academy Press. 1999.

13. Lannin DR, Mathews HF, Mitchell J, Swanson MS, Swanson FH, Edwards MS: Influence of socioeconomic and cultural factors on racial differences in

\author{
Correspondence address: \\ Dr. M. Manoharan \\ Associate Professor, Department of Urology \\ University of Miami Miller School of Medicine \\ P.O. Box 016960 (M814) \\ Miami, Fl 33101, USA \\ Fax: + 1305 243-4653 \\ E-mail:mmanoharan@med.miami.edu
}

\section{EDITORIAL COMMENT}

The authors present a retrospective study including 448 patients with bladder cancer submitted to radical cystectomy. This study examined patterns of invasive bladder cancer by ethnicity and gender. Unfortunately, the article does not discuss socioeconomic factors and other issues such as access to the health care and the pattern of referral from primary care physicians that could influence the indexes analyzed by the study. When the authors compared Hispanic and NHW patients concerning smoking and histological grade, the risks did not differ significantly, indicating that ethnic differences in bladder cancer incidence may not be related to smoking and grade. However, cancer risk may be highly dependent on the number of cigarettes smoked per day and we have not this information in the text. The Hispanic group had a late-stage presentation of breast cancer. JAMA. 1998; 279: 1801-7.

14. Mitchell JL: Cross-cultural issues in the disclosure of cancer. Cancer Pract. 1998; 6: 153-60.

15. Gomez SL, Kelsey JL, Glaser SL, Lee MM, Sidney $\mathrm{S}$ : Inconsistencies between self-reported ethnicity and ethnicity recorded in a health maintenance organization. Ann Epidemiol. 2005; 15: 71-9.

Accepted after revision: September 9, 2008

higher incidence of local recurrence (54\%) compared to $(43 \%)$ in NHW group, although it did not reach statistical significance $(\mathrm{P}=0.355)$. In this preliminary study, Hispanic patients are at greater risk of having higher stage disease at RC, compared to NHW and the disease specific survival of Hispanic patients after $\mathrm{RC}$ is lower compared to NHW. Evaluation of genetic differences between the 2 groups will need to be addressed in future studies.

\section{Dr. Antonio Augusto Ornellas Section of Urology National Institute of Cancer and Hospital Mário Kröeff Rio de Janeiro, Brazil E-mail: ornellasa@hotmail.com}

